\title{
Record of Alteration by Heavy Ices in a Cometary Clast in a Primitive Meteorite
}

Katherine Burgess ${ }^{1}$, Rhonda Stroud ${ }^{2}$, Larry Nittler ${ }^{3}$ and Josep Trigo-Rodriguez ${ }^{4}$

${ }^{1}$ U.S. Naval Research Laboratory, District of Columbia, United States, ${ }^{2}$ U.S. Naval Research Laboratory, United States, ${ }^{3}$ Carnegie Institution of Washington, United States, ${ }^{4}$ Meteorites, Minor Bodies and Planetary Sciences Group, Institute of Space Sciences (CSIC-IEEC), Barcelona, Catalonia, Spain, Catalonia, Spain

Known and probable cometary material available for study on Earth includes chondritic-porous interplanetary dust particles (CP-IDPs), ultra-carbonaceous Antarctic micrometeorites (UCAMMs), and particles returned by the Stardust mission. Recently, a hypothesized cometary micro-xenolith was identified in the Renazzo-like (CR) carbonaceous chondrite LaPaz Icefield (LAP) 02342 [1]. The carbonrich clast is approximately $100 \mu \mathrm{m}$ in diameter and is embedded in the fine-grained matrix of the meteorite. In addition to the very high abundance of carbonaceous material and presolar grains in the clast and distinct isotopic and functional-group nature of the organic matter compared to the matrix and other typical CR matrices, the carbon-rich clast also contains GEMS grains (glass with embedded metal and sulfides) that are similar to those seen in CP-IDPs and UCAMMs [1]. The carbon-rich clast is also enriched in Na compared to the surrounding matrix and contains 16O-poor Na-sulfates in regions that appear to have experienced minor aqueous alteration of some silicates. Such 160-poor material is rare in the sample record and has been observed previously in the matrix of ungrouped carbonaceous chondrite meteorite Acfer 094 [2,3] and in IDPs [4], albeit with different mineralogy. UCAMMs rich in Na (compared to chondritic values) have been reported [5], although a similar sulfate phase has not been identified. The overabundance of $\mathrm{Na}$ in comets compared to the chondritic ratio was initially proposed to be a consequence of preferential depletion of this moderately volatile element in the inner disk, with transfer into the region of condensation of cometary materials [6]. If such scenario is correct, perhaps primordial cometesimals were created in a 160-poor environment. Further study of this clast can help us understand the extent and effects of alteration and potential source of 16O-poor ices.

FIB samples were prepared with an FEI Helios G3 equipped at the Naval Research Laboratory (NRL). No e-beam imaging was done of the sample during thinning in order to avoid changing the functional group chemistry of the organic matter prior to STEM measurements. STEM analysis was performed with the Nion UltraSTEM 200-X at NRL. The microscope is equipped with a Gatan Enfinium ER spectrometer for electron energy loss spectroscopy (EELS) and a windowless, 0.7 sr Bruker SDD-EDS detector. Data were collected at 60 and $200 \mathrm{kV}$.

The sodium-sulfate phase noted in previous work on this clast [1] is part of a complex assemblage that includes two different carbonates and a hydrated clay or carbonate, as well as organic matter with variable functional group chemistry and other altered Fe-bearing silicates with Fe-sulfides (Fig. 1). A large carbonate with small vesicles or other low-density phase contains $\mathrm{Fe}, \mathrm{Mg}, \mathrm{Mn}$, and $\mathrm{Ca}$, similar to ankerite. A second Na-Ca-carbonate phase, identified by the large carbonate peak in C-K EELS (290.4 eV) is intimately mixed with a phase that also exhibits an additional C-K peak at $287.4 \mathrm{eV}$ for aliphatic C (Fig. 2f). Silicon is seen in EDS maps of the same region, and EELS shows peaks at 8.6 and $13.5 \mathrm{eV}$ associated with the presence of water or hydrogen [7], suggesting clay is present [8]. The Na-sulfate is highly beam sensitive, and several grains of Na-sulfate show large peaks at 3.4 and $5.8 \mathrm{eV}$ (Fig. 2e).

The organic matter $(\mathrm{OM})$ near the carbonates and sulfate contains 5-8 at\% $\mathrm{Na}$ along with significant $\mathrm{O}$ (21-28 at\%). Nitrogen (2-4 at\%), $\mathrm{Cl}(0.5-1$ at $\%), \mathrm{F}(0-0.4$ at $\%)$, and $\mathrm{S}(0.3-2$ at $\%)$ are also present and 
quite variable. Measured at $60 \mathrm{kV}$, the C-K EELS edge shows distinct peaks at 285, 286.4, 287.7, and 289 $\mathrm{eV}$, although relative peak heights can vary significantly along with chemistry in different areas. Small grains 40-60 $\mathrm{nm}$ across show large enrichments in $\mathrm{Cl}$ and $\mathrm{F}$, possibly in $\mathrm{NaCl}$ and $\mathrm{MgF}$, respectively.

Further analysis and understanding of the phases present in the Na-rich region of the hypothesized cometary xenolith will provide a better understanding of the reactions that have taken place in this grain and the nature of the 160-poor phase(s). The possible reactions of cometary material with water derived from heavy, Na-rich cometary ices to form the assemblage seen here can help constrain the degree of the alteration, conditions during alteration, and the fluid chemistry required.

Acknowledgements

This work was supported by NASA award 80HQRT19T0038 to RMS.

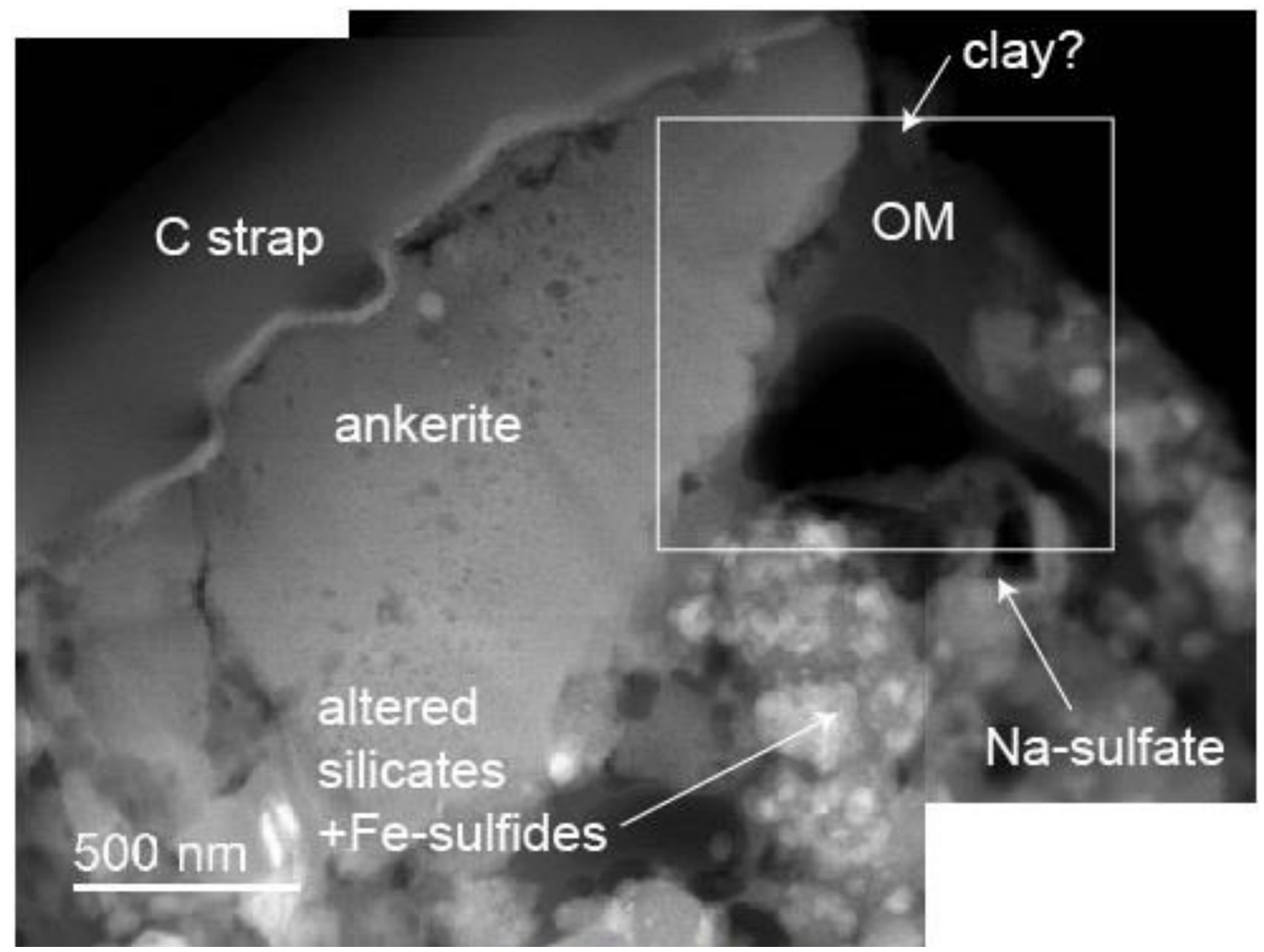

Figure 1. HAADF image of Na-rich phases from the altered rim of the cometary clast. Box indicates location of images in Fig. 2. 

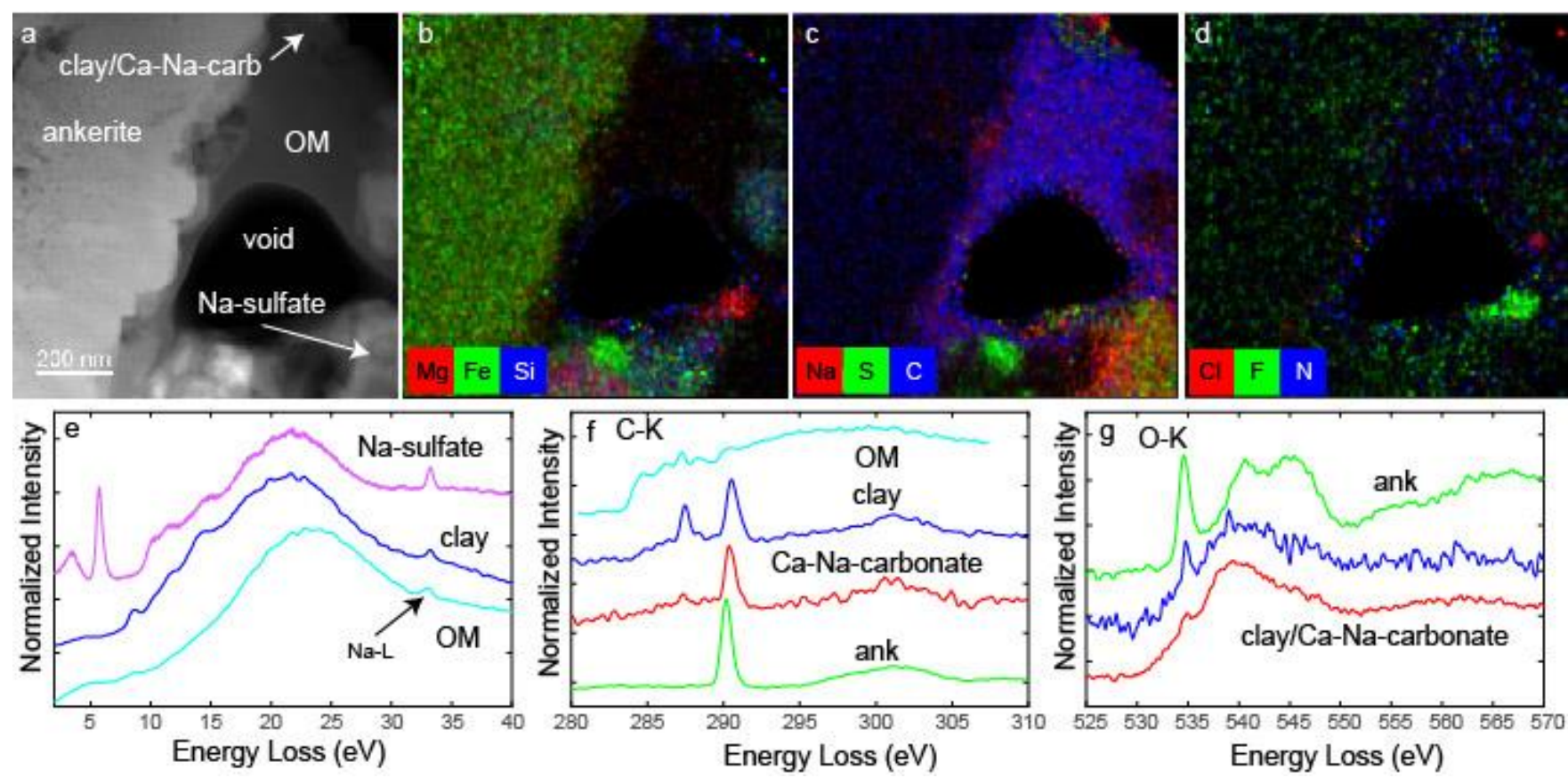

Figure 2. (a) HAADF image and (b-d) EDS maps showing phases within the Na-rich region of the carbonrich clast. (e) Low-loss (f) C K-edge and (g) O K-edge EELS showing features from the phases of interest including the organic matter (OM), ankerite (ank), Na-sulfate, Na-Ca-carbonate, and possible clay.

\section{References}

1 Nittler, L. R. et al. A cometary building block in a primitive asteroidal meteorite. Nat. Astron. 3, 659666, doi:10.1038/s41550-019-0737-8 (2019).

2 Sakamoto, N. et al. Remnants of the early Solar System water enriched in heavy oxygen isotopes. Science 317, 231-233, doi:10.1126/science.1142021 (2007).

3 Seto, Y. et al. Mineralogical characterization of a unique material having heavy oxygen isotope anomaly in matrix of the primitive carbonaceous chondrite Acfer 094. Geochim. Cosmochim. Acta 72, 2723-2734, doi:10.1016/j.gca.2008.03.010 (2008).

4 Starkey, N. A., Franchi, I. A. \& Lee, M. R. Isotopic diversity in interplanetary dust particles and preservation of extreme 160-depletion. Geochim. Cosmochim. Acta 142, 115-131, doi:10.1016/j.gca.2014.07.011 (2014).

5 Yabuta, H. et al. Formation of an ultracarbonaceous Antarctic micrometeorite through minimal aqueous alteration in a small porous icy body. Geochim. Cosmochim. Acta 214, 172-190, doi:10.1016/j.gca.2017.06.047 (2017).

6 Trigo-Rodríguez, J. M., Llorca, J. \& Fabregat, J. Chemical abundances determined from meteor spectra - II. Evidence for enlarged sodium abundances in meteoroids. Mon Not R Astron Soc 348, 802810, doi:10.1111/j.1365-2966.2004.07389.x (2004).

7 Bradley, J. P. et al. Detection of solar wind-produced water in irradiated rims on silicate minerals. Proc. Nat. Acad. Sci. 111, 1732-1735, doi:10.1073/pnas.1320115111 (2014).

8 Garvie, L. A. J. \& Buseck, P. R. Prebiotic carbon in clays from Orgueil and Ivuna (CI), and Tagish Lake (C2 ungrouped) meteorites. Meteor. Planet. Sci. 42, 2111-2117, doi:10.1111/j.19455100.2007.tb01011.x (2007). 